\title{
Research on feature extraction and segmentation of rover wheel imprint
}

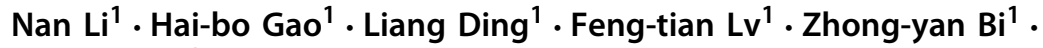 \\ Yi-da Wang ${ }^{1}$
}

Published online: 31 July 2018

(c) The Author(s) 2018

Keywords Rover - Trace imprint $\cdot$ Mechanism analysis $\cdot$ Feature extraction $\cdot$ Feature recognition

\section{Introduction}

In deep space exploration mission based on rovers, the information collected by sensors need to be adequately analyzed and utilized. The wheel imprint photograph of the rover contains important information such as wheel motion parameters, soil characteristic parameters and movement status of the rover [1]. Non-contact methods are commonly been used to measure wheel imprint, using instruments such as on-board camera [2] and laser rangefinder [3]. The method of using laser rangefinder to collect wheel imprint information is not restricted by the illumination condition, thus highly reliable but more additional instrument weight will be added to the rover. The measurement result of on-board camera method can be affected by the illumination condition and is relied on later stage image processing, yet no additional instrument weight means better cost performance and engineering practicability than the previous one.

However, the premise of collecting wheel imprint information is wheel imprint area segmentation. Common segmentation methods are usually based on gray histogram feature, edge characteristics and regional characteristics regardless of unique frequency domain characteristics and morphological characteristics of wheel imprint. These general methods are mostly relied on the pixel distribution model of image which can be utilized to all kinds of images, and wheel imprint formation mechanism model is not considered; therefore, the results are not accurate. Understanding the mechanism of wheel imprint formation, abstracting the morphology of wheel imprint

Nan $\mathrm{Li}$

linan0015@gmail.com

1 State Key Laboratory for Robotics and System, Harbin Institute of Technology, Harbin 150001, China 
and the frequency domain characteristics can help to improve the segmentation precision and efficiency of wheel imprint image.

Image segmentation can be divided into two steps: characteristics analysis and characteristics classification. In the aspect of characteristics analysis, the quality of characteristics recognition will directly affect the results of segmentation, so a large number of scholars have carried out related researches. The initial texture analysis is based on the first- or second-order statistical methods. Haralick and others proposed a gray-level co-occurrence matrix in 1973 and extracted 14 values as texture characteristics for image classification [4]. On this basis, more characteristics recognition methods such as grayscale difference statistical [5], gray range matrix [6], emerged. Subsequently, Markov random fields (MRF) [7], Gauss Markov random field (GMRF) [8] and Gibbs random field model [9] are applied to extract texture characteristics. In 1980, Laws [10] proposed a local texture energy extraction method to analyze texture characteristics. In recent years, multi-resolution and multi-channel texture analysis methods such as Gabor transform and wavelet transform have attracted wide attention and been researched [11], and the local two valued mode (LBP) proposed by Ojala et al. [12] is the most representative research achievement in recent years. In terms of characteristics classification, no-learning clustering algorithm has been commonly used. Macqueen's [13] $K$ mean value clustering algorithm proposed in 1967 and Dunn's [14] fuzzy $C$ mean value clustering algorithm (FCM) based on fuzzy theory are in common use. Influenced by the development of artificial intelligence, researchers put forward a classification method based on artificial neural network. The basic idea is to train the neural network with samples and then use the trained neural network to do image segmentation [15]. In 1995, the support vector machine (SVM) classification method proposed by Cortes et al. [16] is also a classification method based on machine learning.

In this paper, a new wheel imprint image segmentation method is proposed based on wheel imprint formation mechanism. The contents of the study are as follows:

1. Researches analyzing and extracting characteristics from wheel imprint images are carried out. By analyzing wheel-ground interaction mechanism on planet surface, a wheel imprint morphology model is established. Based on the morphology model, the morphology, cycle and orientation characteristics of wheel imprint can be extracted, and the cycle characteristic and orientation characteristic model can be established.

2. Researches of image segmentation methods are carried out. The image enhancement method is used to preprocess the wheel imprint images which were taken under poor illumination conditions. The eigenvectors are constructed based on the periodic and directional characteristics model of the wheel imprint. The clustering algorithm is used to classify the eigenvectors, and the result of which is used to segment wheel imprint image.

3. Experimental verification of wheel imprint image segmentation methods under various rover working conditions including different side angle and slip rate. 


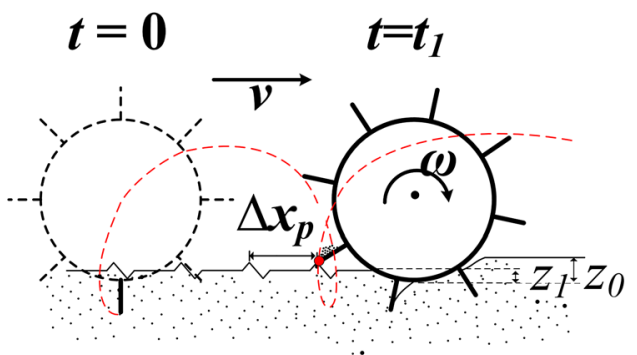

- Point P - - - Trajectory of Point P

(a)

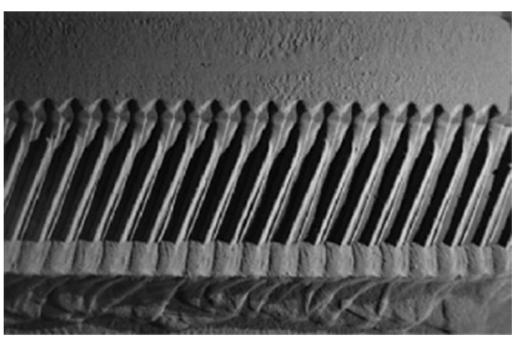

(b)

Fig. 1 Periodicity and directionality of wheel imprint. a Forming mechanism of wheel imprint and $\mathbf{b}$ wheel imprint photograph

\section{Analysis and extraction of wheel imprint image characteristics}

\subsection{Brief introduction of wheel imprint formation}

The wheel imprint of a rover is the indentation left by wheel thorns when it passes the planet surface. As shown in Fig. 1a, when the rover wheel interacts with soil, the thorn cycloid have two intersection points with soil surface, respectively, the contact and separation point. Wheel imprint forms adjacent to the separation point. When the wheel rolls a cycle, each thorn around it produces a wheel imprint unit. If the number of thorn is $N, N$ wheel imprints will be formed in a cycle. When the rover wheel slip rate remains unchanged, distance between wheel imprint units $\Delta x_{p}$ is a constant, and the wheel imprint has a certain periodicity on the soil surface.

The equation of wheel imprint produced by a $n_{L}$-thorn wheel is as follows:

$$
x_{p n}^{0}(j)=r\left(\frac{2 \pi j}{n_{L}}-\theta\right)(1-s)+\sin (2 \pi j-\theta) \quad j=1,2,3 \ldots
$$

$j$ is the number of thorn already interacted with the ground, $r$ is the wheel radius, $n_{L}$ is the number of wheel imprint units generated by each rotation of the wheel, $s$ is the slip rate, and the angle of thorn when it leaves the ground is $\theta$. Equation (1) is a periodic function, and the interval between the two adjacent wheel imprint is as follows:

$$
\Delta x_{p}=x_{p}^{0}(j+1)-x_{p}^{0}(j)=\frac{2 \pi(1-s) r}{n_{L}}
$$

When the wheel's rolling direction is inconsistent with the driving direction of the rover (the wheel has a side slip angle), wheel imprint will still show a consistent direction, as shown in Fig. 1 b. 


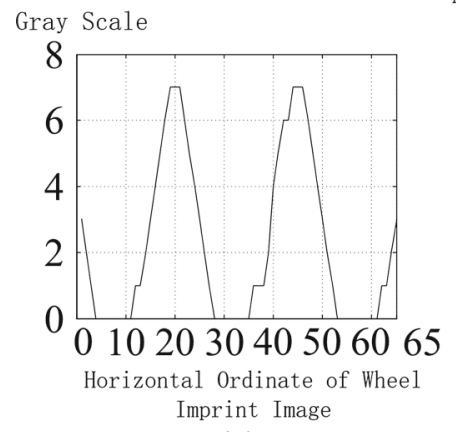

(a)

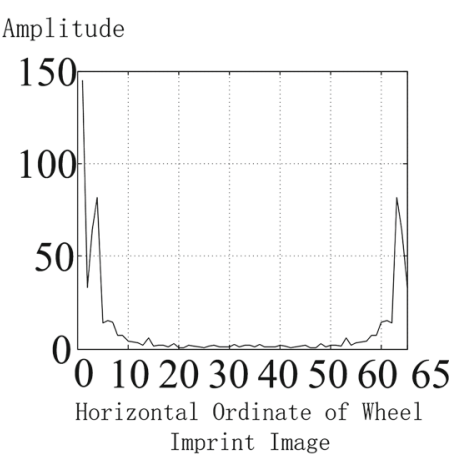

(b)

Fig. 2 Extraction of periodic feature of wheel imprint. a Change in gray scale and $\mathbf{b}$ result of Fourier spectrum

\subsection{Analysis and extraction of periodic characteristics}

According to the mechanism of wheel imprint formation, wheel imprint is periodically composed of concave area and convex area, and they are interlaced. Therefore, onedimensional Fourier transform can be used to analyze the frequency information of a single pixel on a sampling line or a number of pixels adjacent to the sampling line. In order to analyze the frequency domain characteristics of wheel imprint, images are sampled along the moving direction of the rover. As the image is discrete, the position coordinates of the pixels on the line need to be obtained by the point to point comparison interpolation method.

After obtaining the coordinate locations of these pixels, the gray values of them are arranged in sequence according to their value on $X$ coordinates from small to large, and a one-dimensional array $f(x)$ is obtained, then one-dimensional discrete Fourier transform is used toward $f(x)$ :

$$
F(f(x))=F(u)=\sum_{x=0}^{N-1} f(x) \mathrm{e}^{-\frac{j 2 \pi u x}{N}}
$$

The spectrum analysis of the array is carried out. Its maximum amplitude in frequency spectrum, namely the amplitude of the zero frequency, corresponds to the DC component, and the frequency corresponding to the second largest amplitude is the main frequency. Therefore, the fundamental frequency $f_{n}$ in the spectrum is used as the eigenvalue of the pixel. Figure $2 \mathrm{a}$ shows the change in gray value along rover moving direction in a wheel imprint area. Figure $2 b$ is the Fourier transformation amplitude graph of the wheel imprint image.

\subsection{Analysis and extraction of directional characteristics}

As shown in Fig. 1b, wheel imprint has unequivocal directionality; thus, the image can be transformed from space domain to frequency domain by two-dimensional discrete 
Fig. 3 Spectrum of imprint image

Fourier transform; then, the direction of wheel imprint texture can be analyzed in frequency domain. The two-dimensional discrete Fourier transform equation for the image is shown as follows:

$$
F(f(x, y))=F(u, v)=\sum_{x=0}^{M-1} \sum_{y=0}^{N-1} f(x, y) \mathrm{e}^{-j 2 \pi\left(\frac{u x}{M}+\frac{v y}{N}\right)}
$$

In the above formula, $M, N$ represents the width and height of the image, respectively, and $f(x, y)$ is the gray value at point $(x, y)$.

Figure 3 is the two-dimensional Fourier transform spectrum of Fig. 1b, and the transformation equation is:

$$
\begin{aligned}
F(f(x, y)) & =F(u, v) \\
& =\sum_{x=-\frac{M-1}{2}}^{\frac{M-1}{2}} \sum_{y=-\frac{N-1}{2}}^{\frac{N-1}{2}} f(x, y) \exp \left(-i 2 \pi\left(\frac{u x}{M}+\frac{v y}{N}\right)\right)
\end{aligned}
$$

The direction of the wheel imprint texture corresponds to the spectral line with maximum energy in the spectrum, namely the direction of the brightest line. In a $M * N$-pixel photograph of wheel imprint, the expression of line segment is:

$$
\left\{\begin{array}{l}
y=a x+b \\
F(x, y)=c
\end{array}\right.
$$

$a, b$ and $c$ of the Eq. (6) are constant. $a$ is the slope of the line; $b$ is the intercept of the line; $c$ is the modulus of the Fourier transform at $(x, y)$. Two end points of the line segment are $\left(x_{1}, y_{1}\right),\left(x_{2}, y_{2}\right)$, respectively.

$$
\begin{aligned}
|F(u, v)|^{2}= & {\left[\sum_{x=0}^{M-1} c \cos \left(2 \pi\left(\frac{x u}{M}+\frac{(a x+b) v}{N}\right)\right)\right]^{2} } \\
& +\left[\sum_{x=0}^{N-1} c \sin \left(2 \pi\left(\frac{x u}{M}+\frac{(a x+b) v}{N}\right)\right)^{2}\right]^{2}
\end{aligned}
$$


Transform Eq. (7) into linear integral format, and let:

$\alpha=\frac{2 \pi(N u+M v a)}{M N}, \gamma=\frac{\alpha}{2}\left(x_{2}-x_{1}\right)$, the equation can be converted into:

$$
\begin{aligned}
|F(u, v)|^{2} & =\frac{c^{2}}{\alpha^{2} M N}\left[2-2 \cos \alpha\left(x_{2}-x_{1}\right)\right] \\
& =\frac{c^{2}\left(x_{2}-x_{1}\right)^{2}}{M N} \frac{\sin ^{2} \gamma}{\gamma^{2}}
\end{aligned}
$$

When $\sin \gamma \leq \gamma$ :

$$
\lim _{\gamma \rightarrow 0} \frac{\sin ^{2} \gamma}{\gamma^{2}}=1
$$

This means when $\gamma \rightarrow 0, F(u, v)$ reaches its maximum value, namely when:

$$
\gamma=\frac{\alpha}{2}\left(x_{2}-x_{1}\right)=\frac{\pi(N u+M v a)}{M N}\left(x_{2}-x_{1}\right)=0
$$

It can be concluded from the result that the relation between the slope $(k)$ of the brightest line in the Fourier transform spectrum and the slope $(a)$ of the wheel imprint texture direction is as follows:

$$
a=-\frac{N}{k M}=-\frac{u N}{v M}
$$

In Eq. (6), $M, N$ is the width and height of the image, respectively. It can be deducted that the slope $(k)$ of the brightest line is only related to the slope $(a)$ of the texture, so the direction angle of the wheel imprint texture can be obtained by analyzing the Fourier energy spectrum.

Figure 3 is spectrum of imprint image. The spectrum image in Cartesian coordinates is mapped to the energy angle distribution in polar coordinates, so as to extract the slope of this line conveniently. Figure 4a shows calculation process of angular energy distribution. In this paper, the energy spectrum is analyzed by means of the radiation scanning method. The direction angle of the brightest spectral line can be obtained by analyzing the angular energy distribution of the energy spectrum, mathematical expression of which can be shown as follows:

$$
P(\theta)=\sum_{\theta<\arctan \frac{u}{v}<\theta+\Delta \theta}|F(u, v)|^{2}
$$

In order to get the precise angle of the wheel imprint texture, the angular resolution $\Delta \theta$ is set to be $1^{\circ}$. Since the energy spectrum is centrally symmetric, it is only necessary to add up the angular energy distribution at each angle of the first and second quadrants, respectively, regardless of the third and fourth quadrants.

Figure $4 \mathrm{~b}$ is the angular energy distribution of the spectrum. From the graph, we can see that the angular energy distribution curve has three peaks in the angular direction of $0^{\circ}, 90^{\circ}$ and $168^{\circ}$, among which the sum of the energy in the $168^{\circ}$ directions is 


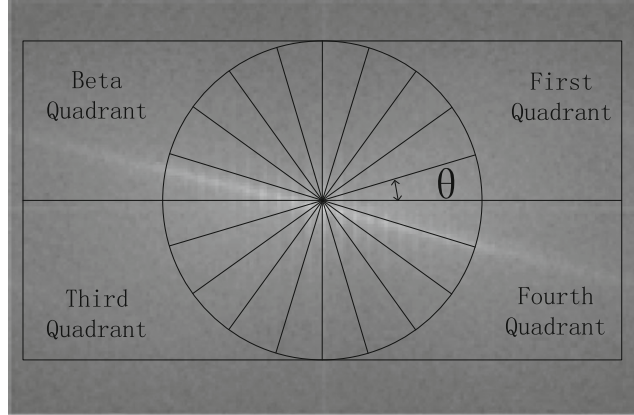

(a)

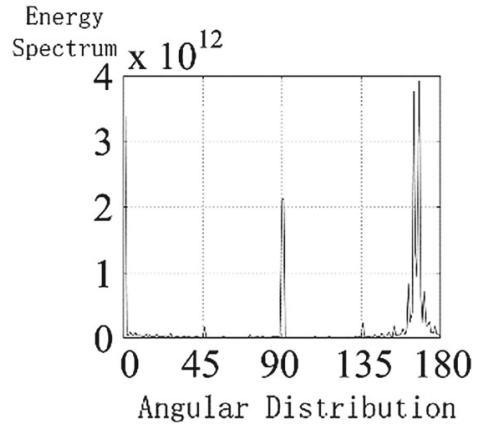

(b)

Fig. 4 Analysis of imprint texture angle. a Calculation of angular energy distribution and $\mathbf{b}$ the angular distribution of the energy spectrum

the largest. This means the angular direction of the brightest line is $168^{\circ}$. Through calculating Eq. (11), the direction angle of the wheel imprint texture is supposed to be $72^{\circ}$.

In order to realize the automatic extraction of the direction characteristics of wheel imprint, two-dimensional Gabor filter is used, mathematical expressions of which is shown below:

$$
\begin{aligned}
h\left(x, y, \theta, f, \sigma_{x}, \sigma_{y}\right)= & \frac{1}{2 \pi \sigma_{x} \sigma_{y}} \\
& \cdot \exp \left\{-\frac{1}{2}\left[\left(\frac{x_{\theta}}{\sigma_{x}}\right)^{2}+\left(\frac{y_{\theta}}{\sigma_{y}}\right)^{2}\right]\right\} \exp \left(j 2 \pi f x_{\theta}\right)
\end{aligned}
$$

In the above formula, $x_{\theta}=x \cos \theta+y \sin \theta, y_{\theta}=y \cos \theta-x \sin \theta, \theta$ is the direction parameter of the filter, $\sigma_{x}$ and $\sigma_{y}$ are the standard deviation of the Gauss envelope on the $X$ and $Y$ axes, respectively, and $\mathrm{f}$ is the frequency of sinusoid. The Gabor response value at the $(X, Y)$ point on the image is:

$$
\begin{aligned}
g\left(X, Y, \theta, \sigma_{x}, \sigma_{y}\right)= & \sum_{x=-u / 2}^{u / 2-1} \sum_{x=-u / 2}^{u / 2-1} I(X+x, Y+y) \\
& \cdot h\left(x, y, \theta, \sigma_{x}, \sigma_{y}\right)
\end{aligned}
$$

$I(X+x, Y+y)$ in the equation is the gray value of the gray image at point $(X, Y)$.

According to Eq. (13), in order to use a Gabor filter, we need to determine 3 parameters, namely the direction parameter $\theta$, the sine wave frequency $f$ and the standard deviation of Gauss envelope $\sigma_{x}$ and $\sigma_{y}$. The wheel imprint texture has consistent direction and the frequency of which will change with the slip rate of the rover wheels, so in this paper we use four Gabor filters with same directional parameters but different sine wave frequency to obtain a response diagram and use the diagram to analyze the features of wheel imprint image. The direction parameter $\theta$ is the wheel imprint 


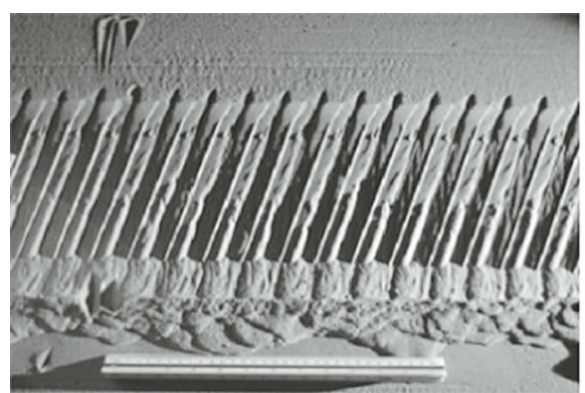

(a)

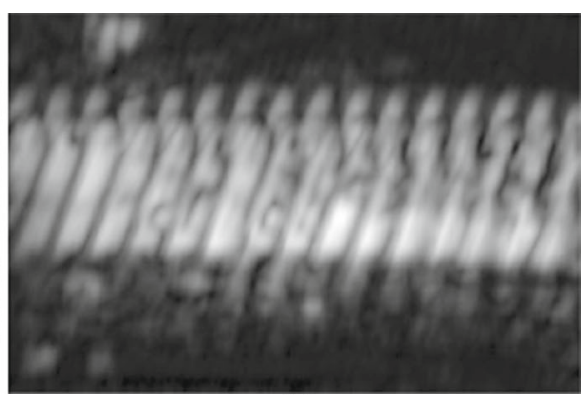

(b)

Fig. 5 Gabor feature of wheel imprint. a Wheel imprint photograph and $\mathbf{b}$ Gabor response graph of wheel imprint

texture direction angle. It can be obtained through Fourier spectrum analysis. The standard deviation $\sigma_{\mathrm{x}}$ and $\sigma_{y}$ can be used to calculate f according to document [17]. Then the Gabor response diagram of wheel imprint image regards to $\theta, \sigma_{\mathrm{x}}$ and $\sigma_{y}$ can be plotted.

As can be seen from Fig. 5b, the Gabor response value of the wheel imprint texture is intense which presents gray in the response graph, while the Gabor response value of the sandy soil is smaller and presents black in the image. It is worth noting that the response value of the white ruler below the wheel imprint photograph is very small in the response graph, and almost invisible, which means that the Gabor filter can effectively filter out the interference components and well retain the wheel imprint texture information.

Since the gap between wheel imprint units is huge, only taking the Gabor response value of a single pixel as feature may cause disruption of the wheel imprint area after image segmentation; in this way the segmented image cannot be processed afterward. In order to make the wheel imprint area after segmentation more complete and of higher quality, the average value and standard deviation of the Gabor response value of a single pixel and its adjacent fields are taken as the eigenvalues of a single pixel. The size of the domain window is $w^{*} w$, which requires at least two wheel imprints being included in the window. Therefore, the value of w can be determined according to the image width $n$ and the number $(f)$ of wheel imprints appearing in the image:

$$
w=\frac{n}{f}
$$

The mean value $M$ and the standard deviation SD of the domain window Gabor response value are:

$$
\begin{gathered}
M=\frac{\sum g(x, y)}{w^{2}} \\
\mathrm{SD}=\sqrt{\frac{\sum[g(x, y)-M]^{2}}{w^{2}}}
\end{gathered}
$$


In the above formula, $G(x, y)$ is the Gabor response value at the $(x, y)$ point of the image, and $w$ is the window size.

\section{Wheel imprint recognition and segmentation method based on texture characteristics}

\subsection{Construction of eigenvector}

After analyzing and extracting three features, $M(I, J), \mathrm{SD}(I, J), f_{n}(I, J)$, of all pixels in the wheel imprint image, they are used to construct a eigenvector. $M(I, J), \operatorname{SD}(I$, $J), f_{n}(I, J)$ are the Gabor response mean value, standard deviation characteristics and frequency characteristics, respectively, at point $(I, J)$. However, each feature's dimension, precision and reliability are not the same, combining them into one eigenvector will lead to poor clustering results. In order to make each characteristics have stronger identification, in this paper, we use Relief method to analyze the influence of them in clustering algorithm, calculate and redistribute their weight, then finally reconstruct the eigenvector with respect to the weight.

The Relief algorithm is proposed by Kira and Rendell [18], assuming that the collection of samples is $D\left\{D_{1}, D_{2}, D_{3}\right.$, and $\left.\ldots D_{\mathrm{n}}\right\}$, its algorithm flow is as follows:

1. Randomly select a sample $D_{a}$, of which the original eigenvector is $\left[D_{i 1}, D_{i 2}, \ldots\right.$ $\left.D_{i k}\right]$

2. From the set of the same kind, find the nearest samples of $D_{a}$ as $H$ at the quantity of $z$. From sample sets of different kind, find the nearest sample $M$ at the quantity of $z$.

3. Calculate diffhit $_{k}$ and diffmiss ${ }_{k}$ respectively:

$$
\begin{aligned}
\operatorname{diffhit}_{k}= & \sum_{j=1}^{z} \frac{\left|D_{a k}-H_{j k}\right|}{\max \left(D_{i k}\right)-\min \left(D_{i k}\right)} \\
\operatorname{diffmiss}_{k}= & \sum_{l \neq \operatorname{class}\left(x_{i}\right)} \frac{P(l)}{1-P\left(\operatorname{class}\left(x_{i}\right)\right)} \\
& \cdot \sum_{j=1}^{z} \frac{\left|D_{a k}-M_{j k}\right|}{\max \left(D_{i k}\right)-\min \left(D_{i k}\right)}
\end{aligned}
$$

4. Calculate feature weighting matrix $\lambda$ :

$$
\lambda_{k}=\lambda_{k}-\frac{\text { diffhit }_{k}-\text { diffmiss }_{k}}{z}
$$

The initial clustering has no weight allocation, so $\lambda_{k}$ is set to be 0 . The eigenvector $\left[\alpha \cdot M(i, j), \beta \cdot \operatorname{SD}(i, j), \gamma \cdot f_{n}(i, j)\right]$ is constructed. $\alpha, \beta$ and $\gamma$ are the weights of each characteristics obtained by the Relief algorithm. As in cluster analysis, each sample categories are unknown, so in this paper we first use FCM clustering algorithm to cluster the original eigenvectors. The weights are distributed and eigenvector model 
is re-established according to features of the sample set after clustering and analyzed by Relief method.

\subsection{Wheel imprint identification segmentation method based on clustering of wheel imprint characteristics}

In this paper, Fuzzy $C$-means clustering is used to classify the eigenvectors and segment the wheel imprint area from the image. The basic idea of the FCM algorithm is to find the optimal clustering center $v_{i}$ and the membership function $u_{i k}$ through iteration, making the objective function to reach its minimum value:

$$
J_{m}(U, c)=\sum_{i=1}^{c} \sum_{k=1}^{N} u_{i k}^{m} \cdot\left\|x_{k}-v_{i}\right\|^{2}
$$

Meanwhile the membership function $u_{i k}$ should satisfy the following equation to optimize the segmentation of the image:

$$
\sum_{i=1}^{c} u_{i k}=1
$$

$m$ is the weight factor, $X_{i}$ is the data sample, $N$ is the total number of data samples and $C$ is the number of clustering categories. Using FCM algorithm, we need to identify two parameters, that is, the number of clustering categories $C$ and the weight factor $\mathrm{m}$. Since we only want to segment the wheel imprint area from the image, the number of clusters $C$ is determined to be 2 . According to the theory from another document [19], weight factor $M$ is also set to be 2 . After determining the parameters of FCM, the data samples consisting of eigenvectors are input into this algorithm. The algorithm can automatically accomplish classification tasks without monitoring and presetting clustering centers.

After finishing the classification, we use the two-value image morphological operation to post-process the classified images, hence eliminating the small erroneous segmentation area, and to smooth the intersection line between wheel imprint area and other area on the classified images. Figure $6 \mathrm{a}$ is the original picture, Fig. $6 \mathrm{~b}$ is a classified image after the morphological processing, and Fig. $6 \mathrm{c}$ is the result of the segmentation.

\subsection{Segmentation process}

To make a conclusion, the wheel imprint image segmentation algorithm proposed in this paper can be divided into three steps:

1. Image preprocessing eliminating unrelated and secondary information through image correction and enhancement algorithm, and strengthening the detectability of characteristic. 


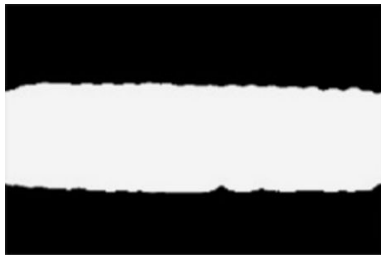

(a)
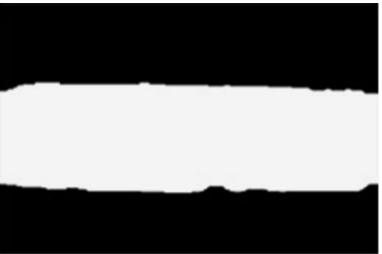

(b)

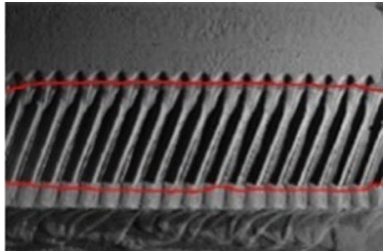

(c)

Fig. 6 Segmentation of wheel imprint. a Original classification, b morphological treatment and $\mathbf{c}$ segmentation results

Fig. 7 Flowchart of algorithm

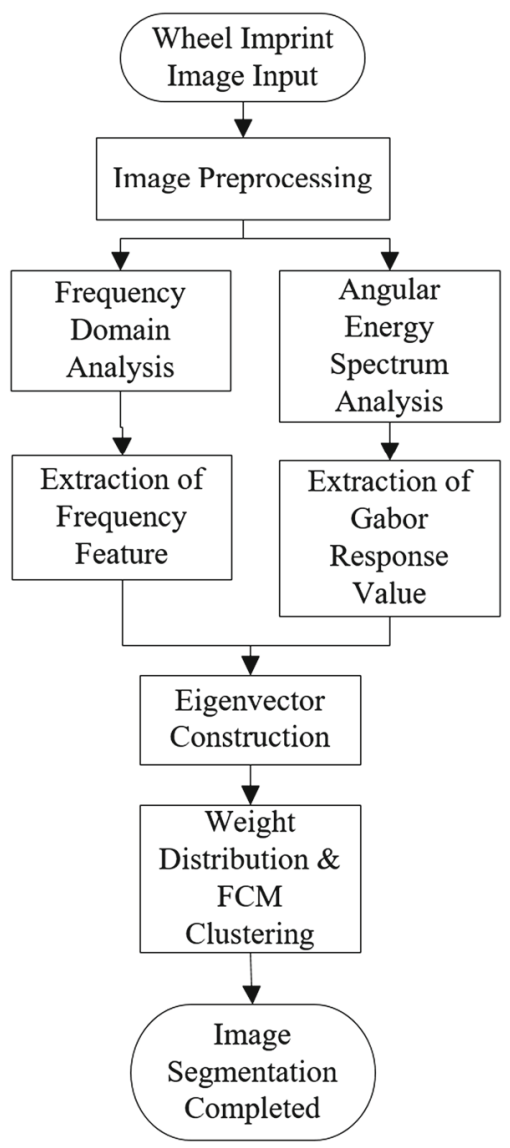

2. Characteristics extraction the characteristics of wheel imprint are abstracted according to the mechanism of formation.

3. Clustering segmentation based on the characteristics of wheel imprints, the wheel imprint area and the other area are separated.

Figure 7 is a flowchart of the entire wheel imprint image segmentation algorithm. 


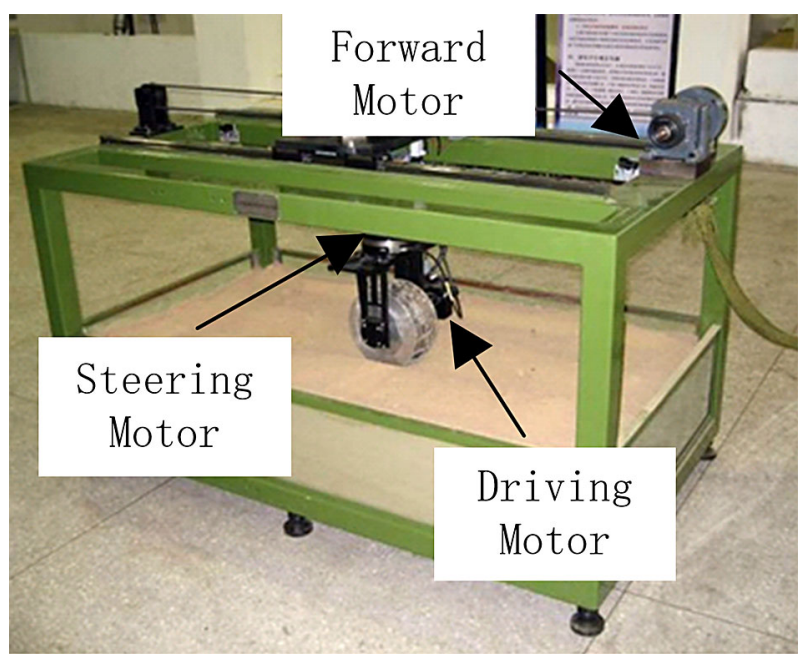

Fig. 8 Wheel measurement system

\section{Experimental verification and result analysis}

\subsection{Design of experimental process}

In order to verify the effectiveness and superiority of this method, an experimental system for the wheel imprint image processing is developed. The system mainly consists of the wheel motion simulation test rig, the machine vision system and the image processing system.

The wheel motion simulation test rig can simulate the moving state of the wheel under different working conditions, thus generating corresponding wheel imprints. As shown in Fig. 8, the wheel motion simulation test rig has a forward motor, a steering motor and a driving motor. Each motor is equipped with a photoelectric encoder to measure the rotation angle of the motor [20]. The driving motor revolves the wheels; the rubber belt driven by the forward motor and the wheel suspension fixed to it are used to simulate the influence of the rover body motion exerting on the wheels, and the steering motor can change the direction angle of the wheels. By controlling the rotational speed of the driving motor and the forward motor, the wheel can move at a desired speed and slip rate. The moving direction of the wheel can be adjusted by changing the turning angle of the steering motor.

In this experiment, the wheel is a cylindrical metal wheel with straight thorns, the radius of which is $140 \mathrm{~mm}$, the wheel width is $150 \mathrm{~mm}$, the number of wheel thorns is 28 and the height of the thorn with respect to the radius direction is $15 \mathrm{~mm}$. The experiment is divided into three steps:

Firstly, wheel imprints are formed through the wheel motion simulation test rig. The wheel motion simulation test rig can simulate the process of the rover wheel motion and produce wheel imprints at desired slip rate and side slip angle. 
Secondly, the wheel imprint image is collected by the visual acquisition system. The visual acquisition system takes digital photographs of wheel imprints and transmits them to the image processing system for processing.

Finally, algorithm proposed in this paper and gray-level co-occurrence matrix algorithm are used to segment the wheel imprint image, respectively, and get the segmentation results under different slip rate and side slip angle condition.

\subsection{Verification of the wheel imprint image segmentation effectiveness}

In order to verify the effectiveness of the method, the segmentation results of the traditional gray-level co-occurrence matrix (GLCM) image segmentation method and the wheel imprint morphological characteristics segmentation method proposed in this paper are compared and analyzed. Table 1 compares the wheel imprint image segmentation results by the gray-level co-occurrence matrix method and the segmentation method proposed in this paper under different side slip angles conditions. Table 2 compares the segmentation results between the gray-level co-occurrence matrix method and the proposed method under different slip rate conditions. According to the comparison between Tables 1 and 2, it can be found that the segmentation method proposed in this paper is more accurate than the gray-level co-occurrence matrix method in the segmentation of wheel imprint area and the other region; furthermore, the erroneous segmentation area is much smaller. 
Table 1 Effect of different algorithm on wheel imprint image processing with different slip angle

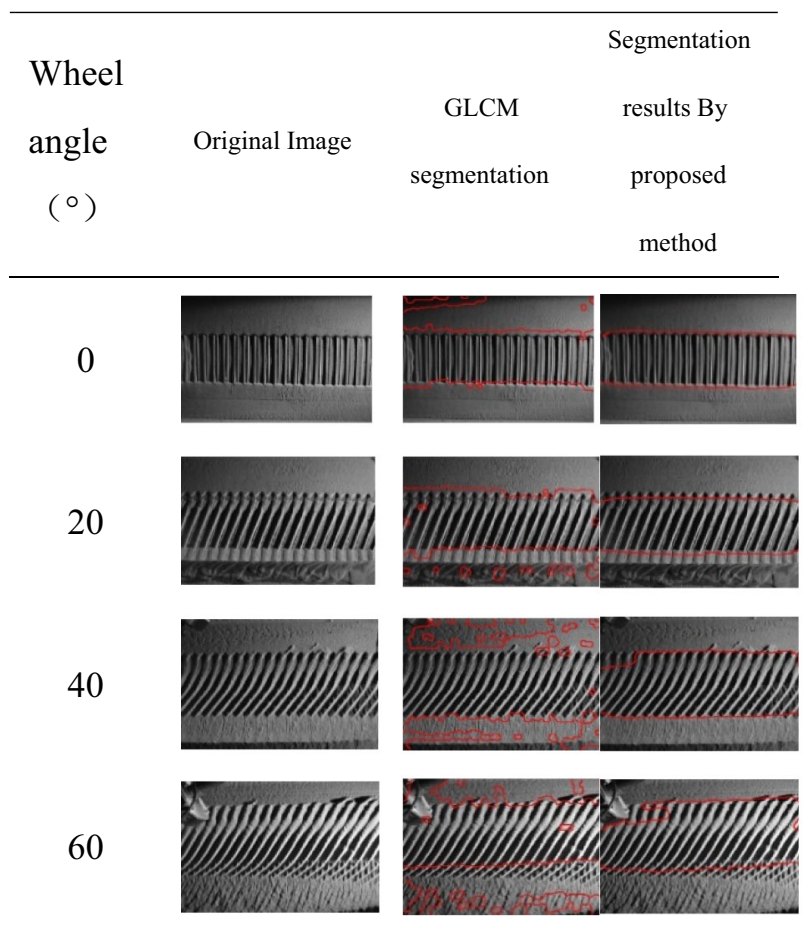

\subsection{Analysis of identification and segmentation results}

In this paper, the segmentation correct rate is taken as the evaluation index, and the segmentation results are evaluated quantitatively. By means of manual segmentation of wheel imprint images, the position of pixels in the wheel imprint area is calibrated. Through statistics, we can get the number of pixels $P_{r}$ in the segmentation results of gray-level co-occurrence matrix and the proposed algorithm, respectively, which are consistent with pixels in the manual segmentation wheel imprint area, and the number of erroneous segmentation pixels $P_{n r}$ which is inconsistent with the manual segmentation results. If the number of pixels in the manual segmentation wheel imprint area is $S$, the segmentation correct rate $T$ of the proposed algorithm will be:

$$
T=\frac{P_{r}}{S}
$$

The segmentation error rate $R$ is:

$$
R=\frac{P_{n r}}{S}
$$


Table 2 Effect of different algorithm on wheel imprint image processing with different slip rate

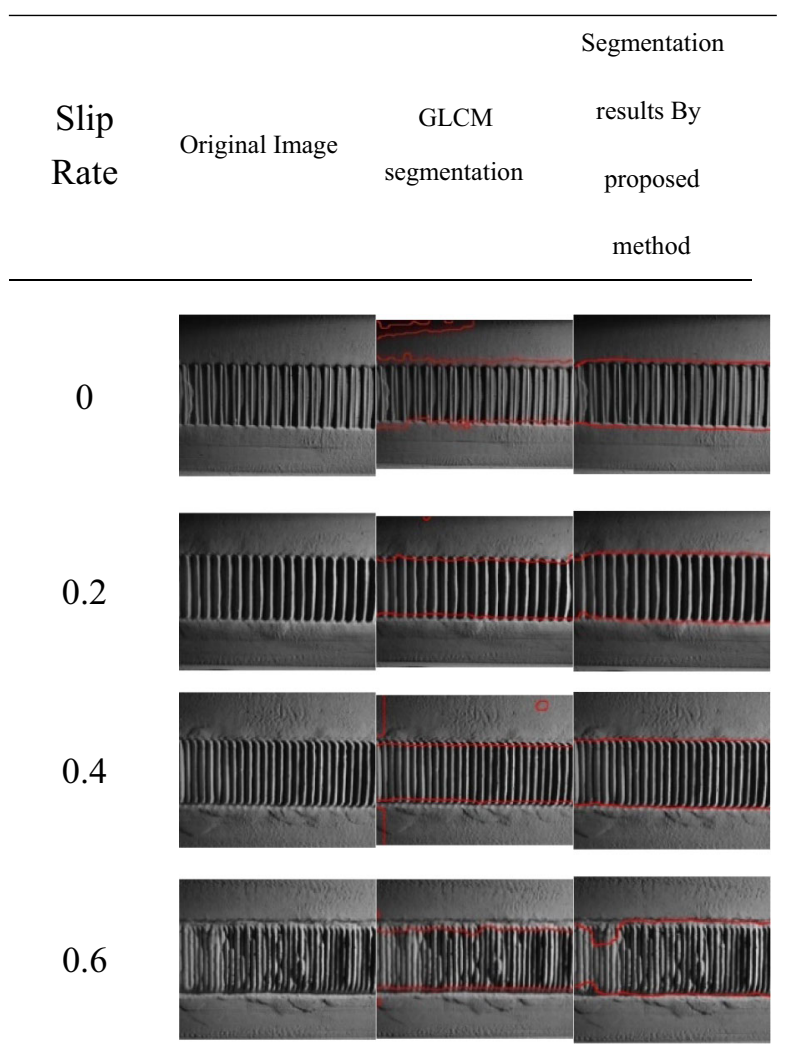

The relative accuracy of the image segmentation algorithm $C$ is defined as:

$$
C=\frac{P_{r}}{P_{r}+P_{n r}}
$$

In these evaluation indexes, the higher the segmentation correct rate $T$, the relative correct rate $C$ and the segmentation error rate $R$ are, the more effective the segmentation algorithm is. Through Formulas (23), (24) and (25), we evaluate the segmentation results of gray-level co-occurrence characteristics segmentation method and method based on wheel imprint texture characteristics in Tables 1 and 2. The sample images were obtained for Table 3 through the design of multi-factor test which is coupled with slippage rate and side angle. Computing hardware is the laptop computer that processes the figure has a CPU of $2.4 \mathrm{GHz}$, and memory of $1.91 \mathrm{~GB}$. Software environment is MATLAB software and functions of Image Processing Toolbox. Then take the mean values of each segmentation correct rate as the algorithm's accuracy, as are shown in Table 3. In addition, the processing speed is also introduced as another evaluation index of each segmentation algorithm. 
Table 3 The correct rate of the division and processing speed

\begin{tabular}{lllll}
\hline $\begin{array}{l}\text { Segmentation } \\
\text { algorithm }\end{array}$ & $\begin{array}{l}\text { Correction rate } \\
(\%)\end{array}$ & Error rate (\%) & $\begin{array}{l}\text { Relative correction } \\
\text { rate }(\%)\end{array}$ & Processing time (s) \\
\hline GLCM & 87.0261 & 22.6936 & 79.4991 & 66.212 \\
$\begin{array}{l}\text { Feature-based } \\
\text { method }\end{array}$ & 87.5069 & 12.7320 & 87.3007 & 17.149 \\
\hline
\end{tabular}

Table 3 shows that the image segmentation method based on the wheel imprint texture has higher correct rate and relative correct rate. The segmentation error rate is lower and the processing speed is about $18 \mathrm{~s}$, which is relatively faster, while error rate of the image segmentation method based on gradient co-occurrence matrix is relatively higher, and the processing speed is slower. The relative correct rate of image segmentation method based on wheel imprint texture characteristics is $7.8 \%$ higher than the method based on gradient co-occurrence matrix, and meanwhile, its processing speed is 3.86 times faster.

It can be concluded from the experiment results that the wheel imprint image segmentation method based on texture characteristics is a more suitable and professional way to segment the wheel imprint area from corresponding images.

\section{Conclusion}

In this paper, the interaction mechanism between the rover wheel and soil ground is analyzed, the inherent morphology of the wheel imprint image and the characteristics of the frequency domain are abstracted, and the eigenvectors are constructed. A wheel imprint eigenvector classification algorithm is proposed based on clustering algorithm. The experiment results show that the wheel imprint area and the other area on the same image can be effectively segmented by this method, and the relative correct rate of the identification and segmentation is $7.8 \%$ higher than the traditional graylevel co-occurrence matrix method. Furthermore, the processing speed of proposed segmentation method is 3.86 times faster.

Open Access This article is distributed under the terms of the Creative Commons Attribution 4.0 International License (http://creativecommons.org/licenses/by/4.0/), which permits unrestricted use, distribution, and reproduction in any medium, provided you give appropriate credit to the original author(s) and the source, provide a link to the Creative Commons license, and indicate if changes were made.

\section{References}

1. Reina G, Ishigami G et al (2008) Vision-based estimation of slip angle for mobile robots and planetary rovers. In: IEEE International Conference on Robotics and Automation. Pasadena, CA, USA, pp 486-491

2. Li N, Gao H et al (2016) Wheel trace imprint image frequency domain analysis and rover wheel slip ratio estimation. J Astronaut 37(11):1356-1364 
3. Jianqiao L, Yang W et al (2013) Non-contact laser measurement of rutting surface on loose ground. Trans Chin Soc Agric Mach 44(7):255-268

4. Haralick RM, Shanmugam K (1973) Textural features for image classification. IEEE Trans Syst Man Cybern SMC-3:610-621

5. Galloway MM (1975) Texture classification using gray level run lengths. Comput Graph Image Process 4:172-179

6. Weszka JS, Dyer CR, Rosenfeld A (1976) A comparative study of texture measures for terrain classification. IEEE Trans Syst Man Cybern SMC-6(4):269-285

7. Cross GR, Jain AK (1983) Markov random field texture models. IEEE Trans Pattern Anal PAMI5(1):25-39

8. Chellappa R, Chatterjee S (1985) Classification of textures using Gaussian Markov random fields. IEEE Trans Acoust Speech Signal Process ASSP-33(4):959-963

9. Derin H, Elliot H (1987) Modeling and segmentation of noisy and textured images using Gibbs random fields. IEEE Trans Pattern Anal PAMI-9:39-59

10. Laws KI (1980) Rapid texture identification. Proc SIPE 238:376-380

11. Arivazhagan S, Ganesan L (2003) Texture segmentation using wavelet transform. Pattern Recogn Lett 24:3197-3203

12. Ojala T, Pietikainen M, Maenpaa T (2002) Multiresolution gray-scale and rotation invariant texture classification with local binary patterns. IEEE Trans Pattern Anal Mach Intell 24(7):971-987

13. Macqueen J (1967) Some methods for classification and analysis of multivariate observations. In: Proceedings of Fifth Berkeley Symposium on Mathematical, vol 1, pp 281-297

14. Dunn JC (1981) Pattern recognition with fuzzy objective function algorithms. Plenum Press, New York

15. Xu X, Ding S et al (2010) New theories and methods of image segmentation. Acta Electron Sin $38(2 \mathrm{~A}): 76-82$

16. Cortes C, Vapnik V (1995) Support-vector networks. Mach Learn 20:273-297

17. Haghighat M, Zonouz S, Abdel-Mottaleb M (2015) CloudID: trustworthy cloud-based and crossenterprise biometric identification. Expert Syst Appl 42(21):7905-7916

18. Kira K, Rendell LA (1992) A practical approach to feature selection. In: Proceedings of the 9th International Conference on Machine Learning. Aberdeen, pp 249-256

19. Bezdek JC (1976) A physical interpretation of fuzzy ISODATA. IEEE Trans Syst Man Cybern SCM6:387-389

20. Liang D, Deng Z, Gao H et al (2015) Interaction mechanics model for rigid driving wheels of planetary rovers moving on sandy terrain with consideration of multiple physical effects. J Field Robot 32(6):827-859 